\title{
Spin-modulated torque waves in ferrimagnetic tunnel junctions
}

\author{
P. Merodio, ${ }^{1, *}$ A. Kalitsov, ${ }^{1,2}$ H. Béa,,${ }^{1}$ V. Baltz,${ }^{1}$ and M. Chshiev ${ }^{1, \dagger}$ \\ ${ }^{1}$ Univ. Grenoble Alpes, INAC-SPINTEC, F-38000 Grenoble, France; CNRS, SPINTEC, F-38000 Grenoble, France; \\ and CEA, INAC-SPINTEC, F-38000 Grenoble, France \\ ${ }^{2}$ MINT Center, University of Alabama, P.O. Box 870209, Tuscaloosa, Alabama 35487-0209, USA
}

(Received 22 April 2014; revised manuscript received 4 November 2014; published 24 December 2014)

\begin{abstract}
Spin-transfer torque (STT) in tunnel junctions with ferromagnetic leads is one of the essential underlying phenomena of modern spintronics. Here, we present a theoretical study of STT in ferrimagnet- (FI-) based tunnel junctions where two FI metal electrodes are separated by a thin nonmagnetic insulating barrier. We show that electronic structure parameters, such as bandwidths and exchange splittings of the FI leads strongly influence STT. In particular, the STT spatial distribution within the leads shows a striking spin-modulated wavelike behavior resulting from the interplay between the exchange splittings of the two FI sublattices. Additionally, we identify the fundamental parameter for quantifying STT characteristic lengths in FI metals, which will also be accessible to experiments, for instance, by ferromagnetic resonance and spin pumping measurements.
\end{abstract}

DOI: 10.1103/PhysRevB.90.224422

PACS number(s): 72.25.-b, 73.23.Ad, 73.40.Gk, 75.50.Gg

The magnetic order of a ferromagnetic (F) thin film can be reoriented by the transfer of angular momentum from a spin-polarized current. This effect, known as spin-transfer torque (STT), was theoretically predicted by Slonczewski [1] and Berger [2] and since then has been the object of extensive investigation due to its applications in spintronic devices [3]. More recently, and despite their vanishing magnetization, similar effects were also proposed for structures containing exclusively antiferromagnetic (AF) metals in which STT was theoretically predicted to act through longer length scales [4]. Essentially, because of alternating moment orientations in ideal AFs, commensurate staggered torques occur generically, and it follows that STT can act cooperatively through the entire AF volume [4]. Over the past few years, a growing number of studies have then considered both theoretical and device aspects of AF-based spintronics [5]. However, AFs are not easy to manipulate experimentally. Alternatively, ferrimagnetic (FI) materials merge characteristic features of both Fs and AFs, namely, a spontaneous macroscopic magnetization together with a partially compensated magnetic structure. As a consequence, combined STT features of Fs and AFs may also occur, giving rise to high local STT values and long-range STT spatial distributions. To verify so, in this paper, we address a theoretical study of the STT spatial distribution in a FI tunnel junction (FI-MTJ) using a tight-binding (TB) model in the framework of the nonequilibrium Keldysh formalism.

Given the layer structure sketched in Fig. 1, the FI-MTJ is described using a single orbital simple cubic TB Hamiltonian which sums the Hamiltonian terms accounting for the isolated left $(L)$ and right $(R)$ electrodes, the barrier $(B)$, and the leadsbarrier interactions,

$$
\hat{H}=\hat{H}_{R}+\hat{H}_{L}+\hat{H}_{B}+\hat{H}_{\mathrm{int}},
$$

\footnotetext{
*pablomerodio@gmail.com

†mair.chshiev@cea.fr
}

where

$$
\begin{aligned}
\hat{H}_{L(R)}= & \sum_{\lambda\left(\lambda^{\prime}\right), \mathbf{k}_{\|}, \sigma}\left(\varepsilon_{\mathbf{k}_{\|}}+\varepsilon_{\lambda\left(\lambda^{\prime}\right)}^{\sigma}\right) \hat{c}_{\mathbf{k}_{\|}, \lambda\left(\lambda^{\prime}\right), \sigma}^{\dagger} \hat{c}_{\mathbf{k}_{\|}, \lambda\left(\lambda^{\prime}\right), \sigma} \\
& +\sum_{\lambda\left(\lambda^{\prime}\right), \mu\left(\mu^{\prime}\right), \mathbf{k}_{\|}, \sigma} t_{\lambda\left(\lambda^{\prime}\right), \mu\left(\mu^{\prime}\right)} \hat{c}_{\mathbf{k}_{\|}, \lambda\left(\lambda^{\prime}\right), \sigma}^{\dagger} \hat{c}_{\mathbf{k}_{\|}, \mu\left(\mu^{\prime}\right), \sigma} \\
\hat{H}_{B}= & \sum_{i, \mathbf{k}_{\|}, \sigma}\left(\varepsilon_{\mathbf{k}_{\|}}+\varepsilon_{i}\right) \hat{c}_{\mathbf{k}_{\|}, i, \sigma}^{\dagger} \hat{c}_{\mathbf{k}_{\|}, i, \sigma}+\sum_{i, j, \mathbf{k}_{\|}, \sigma} t_{i, j} \hat{c}_{\mathbf{k}_{\|}, i, \sigma}^{\dagger} \hat{c}_{\mathbf{k}_{\|}, j, \sigma} \\
\hat{H}_{\mathrm{int}}= & \sum_{\mathbf{k}_{\|}, \sigma}\left(t_{a, \alpha} \hat{c}_{\mathbf{k}_{\|}, a, \sigma}^{\dagger} \hat{c}_{\mathbf{k}_{\|}, \alpha, \sigma}+t_{b, \alpha^{\prime}} \hat{c}_{\mathbf{k}_{\|}, b, \sigma}^{\dagger} \hat{c}_{\mathbf{k}_{\|}, \alpha^{\prime}, \sigma}+\text { H.c. }\right)
\end{aligned}
$$

Here, $\hat{c}_{\mathbf{k}_{\|}, p, \sigma}^{\dagger}$ creates one $s$ electron with spin $\sigma$ on layer $p$ in the Bloch state labeled by the transverse wave number $\mathbf{k}_{\|}$ (translational invariance in the $x z$ plane is assumed through the entire junction). $\varepsilon_{\mathbf{k}_{\|}}$is the in-plane kinetic energy of the Bloch state, and $t_{p, q}$ is the spin-independent hopping matrix element between sites $p$ and $q$. The coupling of the left (right) lead to the barrier is considered through the hopping parameter $t_{a, \alpha}\left(t_{b, \alpha^{\prime}}\right)$ between the first (last) layer of the barrier and the last (first) layer of the left (right) lead.

The spin-dependent on-site energies $\varepsilon_{p}^{\sigma}$ within the leads are split into the $s$-orbital energy $\left(\varepsilon_{0}\right)$ and the magnetic $s$ $d$ exchange interaction between itinerant spins and localized magnetic moments $\left(\Delta_{p}^{\sigma}\right): \varepsilon_{p}^{\sigma}=\varepsilon_{0}+\Delta_{p}^{\sigma}$. We set $\varepsilon_{0}=1.5 \mathrm{eV}$ in both leads and $t=-1 \mathrm{eV}$ in all regions so that all the FIs analyzed here are fully characterized by their on-site dependent $s$ - $d$ interaction $\Delta_{p}^{\sigma}$. Unlike the case of ordinary F-MTJ with a homogeneous exchange splitting value within the electrode, $\Delta_{p}^{\sigma}$ for FI-MTJ here not only alternates in orientation, but also varies in magnitude from one layer to the next one, which defines two different sublattices denoted here as $A$ and $B$ (see Fig. 1). Considering a right FI lead whose first layer next to the $B / F I$ interface is formed by fully uncompensated up spins (sublattice $A$ ), the spin splitting in layer $\lambda^{\prime}$ writes

$$
\Delta_{\lambda^{\prime}}^{\uparrow(\downarrow)}= \begin{cases}-(+) \Delta_{A}, & \text { if } \lambda^{\prime} \text { is odd-sublattice } A, \\ +(-) \Delta_{B}, & \text { if } \lambda^{\prime} \text { is even-sublattice } B .\end{cases}
$$




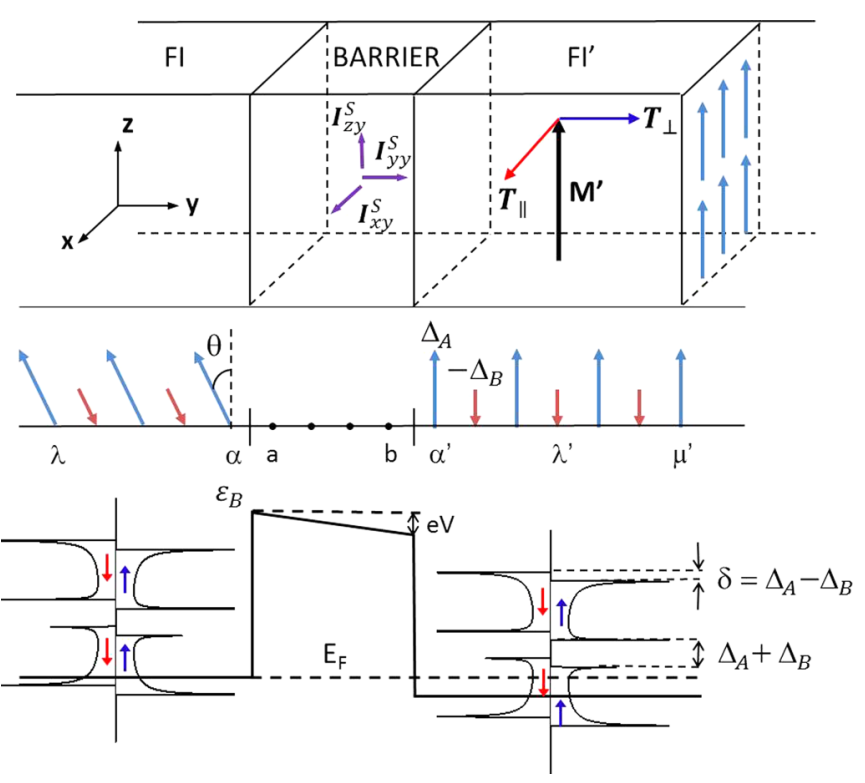

FIG. 1. (Color online) Schemes adapted from Ref. [3] for the specific case of FI leads. (Top) Schematic of the FI-MTJ consisting of left and right semi-infinite FI leads separated by a nonmagnetic barrier of $N_{b}$ atomic layers. The magnetization $M^{\prime}$ of the right lead points along the $z$ direction. The nonequilibrium on-site torques are represented by the in-plane $\left(T_{\|}\right)$and out-of-plane $\left(T_{\perp}\right)$ components, and the spin current densities are also indicated $(I)$. (Middle) Schematic of sublattices $A$ and $B$ in the FI leads with different spin splittings $\Delta_{A}$ and $\Delta_{B}$ corresponding to up and down localized spins, respectively. The magnetization $M$ of the left lead is parallel to the $\mathrm{FI} / B$ interface and is rotated by an angle $\theta$ around the $y$ axis. The greek primed and unprimed letters denote atomic sites in the right and left leads, respectively, and the latin letters denote the sites in the barrier. (Bottom) Schematic of the bands and the potential profile where the 1D densities of states for up and down spins are split by $\delta=\Delta_{A}-\Delta_{B}$ and the lower and upper bands for a given spin are separated by a gap of $\Delta_{A}+\Delta_{B} . \varepsilon_{B}$ is the spin-independent on-site energy in the barrier, and $V$ is the potential applied through the junction. The lower dashed line indicates the Fermi level at equilibrium, set at $0 \mathrm{eV}$ in all regions, when no voltage is applied.

As depicted in Fig. 1, majority and minority bands in the ferrimagnetic leads are split by $\delta=\Delta_{A}-\Delta_{B}$. When an external bias $V$ is applied, the on-site energies inside the insulator are considered to drop linearly with the number of layers (here $N_{b}=3$ ) from $\varepsilon_{b}=5 \mathrm{eV}$ at the $\mathrm{FI} / B$ interface. The parameters modeling the electronic properties of the FI leads constitute a reasonable choice used previously for ordinary F-MTJ based on magnetic transition metals and their alloys [6-8].

The spin densities and spin current mean values are calculated using the density operator directly related to nonequilibrium $2 \times 2$ lesser Green's function in spin space: $\hat{G}_{p, q^{\prime}}^{<\sigma, \sigma^{\prime}}\left(k_{\|}, t\right)=i\left\langle\hat{c}_{k_{\|}, p, \sigma}^{\dagger}(t) \hat{c}_{k_{\|}, q, \sigma^{\prime}}(t)\right\rangle$. Creation and annihilation operators are evaluated in the Heisenberg picture at equal times since the thermal average is calculated in the steady state of the coupled system at room temperature. According to the Keldysh formalism [9] its Fourier transform can be written as $\hat{G}_{p, q}^{<}=\frac{1}{2}\left(\hat{F}_{p, q}+\hat{G}_{p, q}^{a}-\hat{G}_{p, q}^{r}\right)$. The nonequilibrium Keldysh, $\hat{F}_{p, q}$, advanced, $\hat{G}_{p, q}^{a}$, and retarded $\hat{G}_{p, q}^{r}$ Green's functions for the whole coupled system are expressed in terms of the retarded Green's functions $\hat{g}_{p, q}^{r}$ for each uncoupled region at equilibrium by solving a system of quantum kinetic and Dyson equations [8]. For ordinary F-MTJ [8,10,11], the total torque delivered through the entire $\mathrm{F}$ lead can be evaluated using the interfacial spin currents which requires the computation of the lesser Green's functions derived from $\hat{g}_{\alpha, \alpha}^{r}$ and $\hat{g}_{\alpha^{\prime}, \alpha^{\prime}}^{r}$ at the $\mathrm{F} / B$ and $B / \mathrm{F}$ interfaces only due to the rapid decay of torques from the last interface [12]. In contrast, the precise computation of the local STT distribution in the right FI lead addressed in this paper requires the knowledge of all interlayer matrix elements of the Green's function. Here, the retarded Green's functions for the uncoupled leads $\hat{g}_{p, q}^{r}$ are obtained analytically. Such calculation takes into account that a one-dimensional (1D) FI chain corresponds to a lattice without closed loops that can be mapped into a Bethe lattice or Cayley tree which is completely characterized by its number of nearest neighbors $Z=2$ or its connectivity $K=Z-1$. Like Eq. (2), by splitting the Hamiltonian into an unperturbed site-diagonal term plus an off-diagonal perturbation and by using renormalized perturbation expansion [13] the exact Green's function $\hat{g}_{p, q}^{r}$ can be calculated for the Bethe lattice. Given the above precisions and denoting $d$ as the dimensionality of the junction, the spin density and spin current in the right lead are as follows:

$$
\begin{aligned}
\hat{S}_{\lambda^{\prime}} & =\frac{-i \hbar}{2(2 \pi)^{d}} \int \operatorname{Tr}_{\sigma}\left[\hat{G}_{\lambda^{\prime}, \lambda^{\prime}}^{<\sigma, \sigma^{\prime}} \sigma\right] d E d \mathbf{k}_{\|}, \\
\hat{Q}_{\lambda^{\prime}, \lambda^{\prime}+1} & =\frac{t}{2(2 \pi)^{d}} \int \operatorname{Tr}_{\sigma}\left[\left(\hat{G}_{\lambda^{\prime}+1, \lambda^{\prime}}^{<\sigma, \sigma^{\prime}}-\hat{G}_{\lambda^{\prime}, \lambda^{\prime}+1}^{<\sigma, \sigma^{\prime}}\right) \sigma\right] d E d \mathbf{k}_{\|} .
\end{aligned}
$$

The spatial distribution of in-plane and out-of-plane spintransfer torques within the right electrode is extracted from the discrete divergence in the transport direction $y$ of the spin current in each layer of the lead,

$$
\hat{T}_{\lambda^{\prime}}=-\nabla \cdot \hat{Q}=\hat{Q}_{\lambda^{\prime}-1, \lambda^{\prime}}-\hat{Q}_{\lambda^{\prime}, \lambda^{\prime}+1}=\frac{t}{2(2 \pi)^{d}} \int \operatorname{Tr}_{\sigma}\left[\left(\hat{G}_{\lambda^{\prime}, \lambda^{\prime}-1}^{<\sigma, \sigma^{\prime}}-\hat{G}_{\lambda^{\prime}-1, \lambda^{\prime}}^{<\sigma, \sigma^{\prime}}-\hat{G}_{\lambda^{\prime}+1, \lambda^{\prime}}^{<\sigma, \sigma^{\prime}}+\hat{G}_{\lambda^{\prime}, \lambda^{\prime}+1}^{<\sigma, \sigma^{\prime}}\right) \sigma\right] d E d \mathbf{k}_{\|}
$$

Alternatively, since ballistic transport is considered here, torques can also be extracted from the exchange field [14]: $\hat{T}_{\lambda^{\prime}}=\Delta_{\lambda^{\prime}} \hat{z} \times \hat{S}_{\lambda^{\prime}}$, where the exchange field $\Delta_{\lambda^{\prime}}$ is defined as an angular frequency so that the energy required to reverse one spin in layer $\lambda^{\prime}$ is $\hbar \Delta_{\lambda^{\prime}}=\varepsilon_{\lambda^{\prime}}^{\uparrow}-\varepsilon_{\lambda^{\prime}}^{\downarrow}$ [15]. $\Delta_{\lambda^{\prime}}$ is assumed to match the direction of local magnetization in the local spin-density approximation. The $z$ component of torque is zero, and the in-plane and out-of-plane components of the 


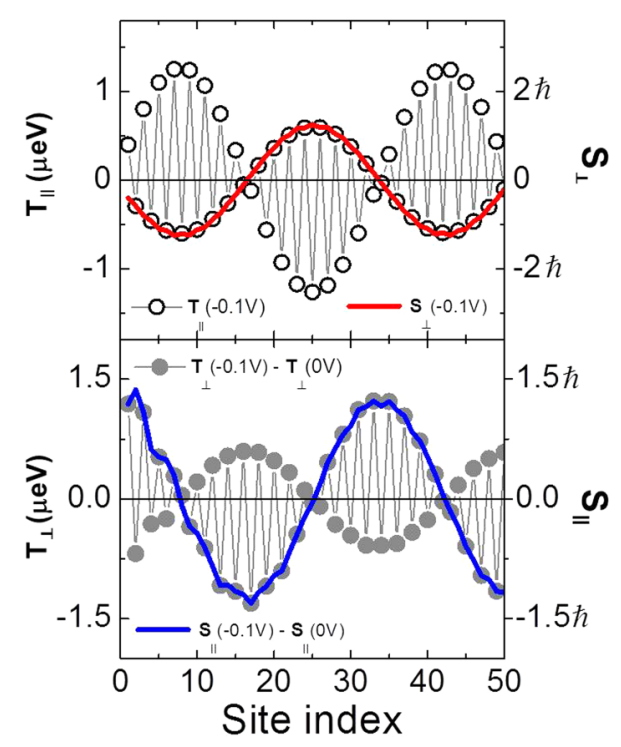

FIG. 2. (Color online) Spatial distribution of the in-plane $\left(T_{\|}\right)$ and out-of-plane $\left(T_{\perp}\right)$ nonequilibrium torque components and local spin-density components $\left(S_{\perp}\right.$ and $\left.S_{\|}\right)$for a $1 \mathrm{D}$ system; $\theta=$ $\pi / 2, \Delta_{A}=0.5, \Delta_{B}=0.25 \mathrm{eV}$, and $V=0.1 \mathrm{~V}$. The equilibrium torques and local spin densities were subtracted: $T_{\|}(0 \mathrm{~V})$ and $S_{\perp}(0 \mathrm{~V})$ equal zero in contrast to $T_{\perp}(0 \mathrm{~V})$ and $S_{\|}(0 \mathrm{~V})$ that result from interlayer equilibrium RKKY interactions. The red and blue curves (symbols) refer to the right- (left-) hand ordinates.

local torques defined in Fig. 1 are given by the so-called spin accumulation in the $x$ and $y$ directions $S_{x}$ and $S_{y}$, respectively: $T_{\lambda^{\prime}}^{\|}=(-1)^{\lambda^{\prime}} \Delta_{\lambda^{\prime}} S_{\lambda^{\prime}}^{y}$ and $T_{\lambda^{\prime}}^{\perp}=(-1)^{\lambda^{\prime}+1} \Delta_{\lambda^{\prime}} S_{\lambda^{\prime}}^{x}$.

In order to illustrate the essential features of the STT spatial distribution in the right FI lead, we first examine a 1D FI-MTJ for which the calculation of local torques does not require the $\mathbf{k}_{\|}$integration. Figure 2 shows the corresponding voltage-induced part of local on-site torques and spin accumulations. The equilibrium zero-bias voltage torques were subtracted. Whereas the in-plane torque is zero at equilibrium $(V=0 \mathrm{~V})$, the out-of-plane torque is not since it accounts for the Ruderman-Kittel-Kasuya-Yosida (RKKY) interaction [16]. Interestingly, both in-plane and out-of-plane torques are staggered and commensurate with the lattice moment orientations. Here, in addition due to the specific FI order of the lead, the torques exhibit a striking wavelike behavior: the FI sublattices $A$ and $B$ host two "torque waves" dephased by half of a period one with respect to the other. Note also that the higher the exchange field, the higher the amplitude of the corresponding torque wave. Figure 2 also demonstrates that the spin-transfer torques calculated using Eq. (8) are perfectly modulated by spin accumulation $\hat{S}_{\lambda^{\prime}}$. Indeed, as explained previously, spin accumulation is calculated independently from Eq. (6), its spatial distribution oscillates exactly at the same period as that of the STT and the ratios $T_{\|} / S_{y}$ and $T_{\perp} / S_{x}$ are constant. This is consistent since, in practice, spin accumulation gives rise to torque waves and can be used as an alternative to calculate the torques. Using spin accumulation thus provides an alternative method for calculating torques not only in ordinary F-MTJ, but also, more generally, in FI- and AF-MTJ.

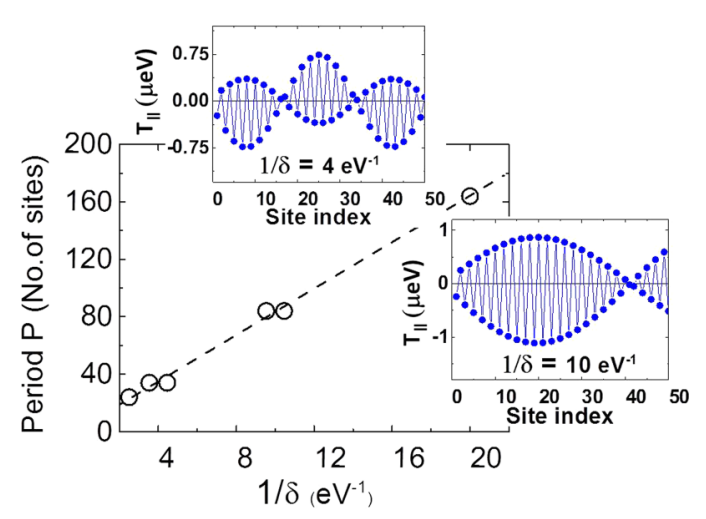

FIG. 3. (Color online) For six different 1D FI leads pairs, $1 / \delta$ dependence of the in-plane torque component period $(P)$ for $\theta=\pi / 2$ and $V=0.1 \mathrm{~V}$. The double symbols at 4 and $10 \mathrm{eV}^{-1}$ represent FI electrodes with unequal spin splittings $\Delta_{A}, \Delta_{B}$ but the same $\delta=\Delta_{A}-\Delta_{B}$. The spin splittings take the following values $\left\{\Delta_{A}, \Delta_{B}\right\}=\{0.5,0.1 ; 0.5,0.25 ; 0.6,0.35 ; 0.5,0.4 ; 0.4,0.3 ; 0.5,0.45\}$. Upper (lower) insets: spatial distribution of the parallel torque wave for $1 / \delta=4 \mathrm{eV}^{-1}\left(1 / \delta=10 \mathrm{eV}^{-1}\right)$. The dashed line is a linear fit to the data.

We next examine the parameters controlling the modulation of the STT. The linear fit in Fig. 3 shows that for a given voltage, the torque wave's period $(P)$ is inversely proportional to the difference $(\delta)$ of magnetic $s$ - $d$ exchange interactions between itinerant spins and localized magnetic moments in each of the two FI sublattices,

$$
P \propto \frac{1}{\delta}
$$

Given the above relation, we point out that the limiting case characterized by $\delta=0$, i.e., when the FI becomes a fully compensated AF, results in a diverging period. This agrees with the $T_{\| \mid}$spatial distribution reported previously in AF-based spin valves [17]. Interestingly, a Taylor expansion at the Fermi energy of the dispersion relation for a 1D FI up to second order in the wave vector recasts relation (9) in terms of the Fermi wave vectors for up and down spins for the case of low band filling: $P \propto \frac{1}{\left(k_{f}^{\uparrow}\right)^{2}-\left(k_{f}^{\downarrow}\right)^{2}}=\frac{1}{\left(k_{f}^{\uparrow}+k_{f}^{\downarrow}\right)\left(k_{f}^{\uparrow}-k_{f}^{\downarrow}\right)}$. This expression is related to the length scales at which torque oscillations decay in conventional F-MTJ from the $B / F$ interface. The two factors $\frac{1}{\left(k_{f}^{\uparrow}-k_{f}^{\downarrow}\right)}$ and $\frac{1}{\left(k_{f}^{\uparrow}+k_{f}^{\downarrow}\right)}$, respectively, are proportional to the voltage-induced and RKKY torque oscillations period in F-based tunnel junctions [18]. Similar to the spatial precession of up and down components of the scattering state in Fs [12], the period $P$ might also be viewed as inversely proportional to the spatial frequency $\Delta k=\left(k_{f}^{\uparrow}-k_{f}^{\downarrow}\right)$ since $\delta$ and $\Delta k$ are proportional to each other with great accuracy for the range of energies considered here.

We also show that the bandwidth of the electronic band structure strongly influences both the torque waves' period and the amplitude of the oscillations. As a result of the TB model used, both upper and lower bandwidths can be parametrized by the hopping $t$, which determines electron mobility inside the whole structure. Figure 4(a) shows the wave period dependence on $t$ for different FI parameters. The two FI represented by crosses and open circles display 

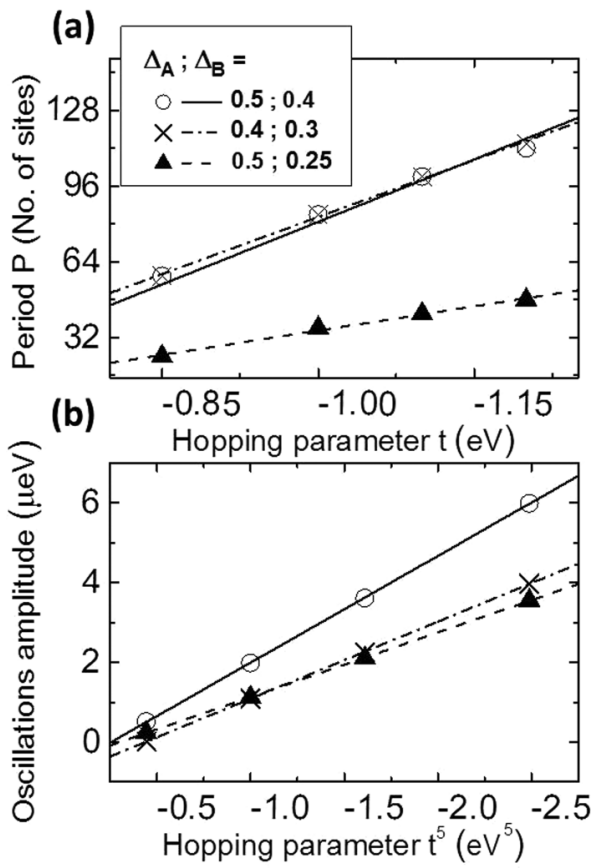

FIG. 4. (a) Dependence with the hopping parameter $(t)$ of the inplane torque component waves period $P$ for $\theta=\pi / 2$ and $V=0.1 \mathrm{~V}$. (b) Corresponding dependence with $t^{5}$ of the torque waves amplitude. The three FI leads of (a) and (b) have $\Delta_{A}$ and $\Delta_{B}$ given in $\mathrm{eV}$ in the caption of (a). The lines are linear fits to the data.

the same period regardless of $t$ since they share the same value of $\delta$ despite unequal spin splittings. The period is however proportional to $t$ in every case and results from the spread of the wave function over more sites for larger values of hopping. The amplitude of the oscillations scales with $t^{5}$ as can be seen in Fig. 4(b); the power factor accounts for the mobility change within the whole structure. It is noteworthy that the hopping parameter does not influence the commensurability between the torques and the lattice moment orientations.

We next analyze the STT local distribution in more realistic 3D FI-MTJ. In this case, the full integration in energy and $\mathbf{k}_{\|}$states is needed, which could potentially extinguish the STT wavelike behavior. However, we found that the torque waves are still present, but the dephased torque contributions introduced by the $\mathbf{k}_{\|}$integration result only in a weak damping that spoils the oscillations' periodic character similar to the case of F-MTJ $[18,19]$. The two insets in Fig. 5 illustrate the in-plane torque deposited in each layer of the right lead in two 3D FI-MTJs with different $\delta^{\prime}$ s. Since damping spoils the periodic character of these oscillations, the concept of period $P$ previously discussed no longer stands. Of note, these damped oscillations cannot be defined as pseudo-oscillations, and we therefore introduce a parameter $\xi$, which keeps track of the wavy length scale. We define $\xi$ as the number of layers between the $B / F I$ interface and the first layer at which the torque wave gets zero, which would correspond to half a period in the 1D case. $\xi$ is plotted in Fig. 5 as a function of $1 / \delta$ which governs the oscillations period in the 1D FI-MTJ. The linear fit provides clear evidence that relation (9) holds for $\xi$ even in three-dimensional (3D) FI-MTJ, thus confirming that the parameter $\delta$ is a key indicator for quantifying STT

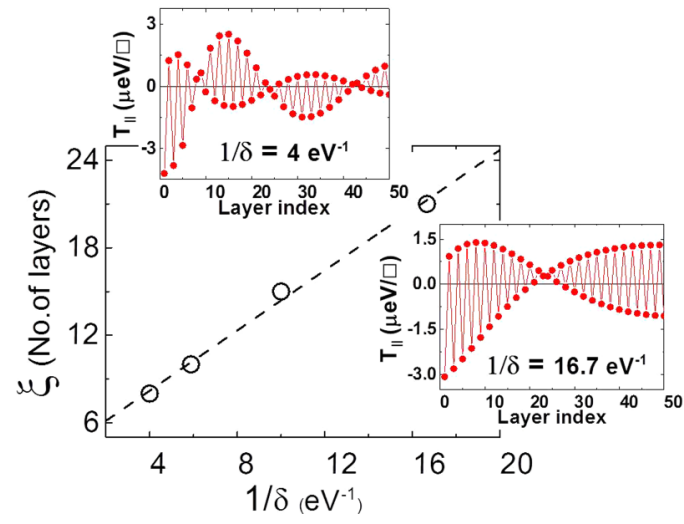

FIG. 5. (Color online) For four 3D FI leads, $1 / \delta$ dependence of the torque characteristic length $(\xi)$ for $\theta=\pi / 2$ and $V=$ $0.1 \mathrm{~V}$. The spin splittings take the following values $\left\{\Delta_{A}, \Delta_{B}\right\}=$ $\{0.5,0.25 ; 0.6,0.43 ; 0.5,0.4 ; 0.4,0.34\}$. Upper (lower) inset: spatial distribution (in $\mu \mathrm{eV}$ per unit area) of the in-plane torque wave for $1 / \delta=4 \mathrm{eV}^{-1}\left(1 / \delta=16.7 \mathrm{eV}^{-1}\right)$. The dashed line is a linear fit to the data.

characteristic lengths in FI metals. We emphasize here the importance of the staggered character of the in-plane torque over the penetration depth given by $\xi$. In effect, the torque deposited in sublattice $A$ adds up to the torque delivered in sublattice $B$ since localized spins owning to different sublattices are aligned in opposite directions. This is in contrast to the case of ordinary F-MTJ where positive and negative local torques are counterbalanced to give the total torque delivered, which is found to be comparable in amplitude to the torques discussed in this paper. Therefore, in-plane torques in FI leads with low values of $\delta$ are expected to be very efficient thus driving current-induced order parameter dynamics.

In conclusion, we carried out a detailed analysis of the STT spatial distribution in FI-based tunnel junctions using a TB Hamiltonian that captures its essential electric and magnetic qualitative characteristics in the framework of the nonequilibrium Keldysh formalism. We find that in-plane and out-of-plane torques are spatially staggered, and they exhibit a striking wavelike behavior perfectly modulated by spin accumulation. Thus, we have addressed in this paper the equivalence of torques computed from the exchange field along the $z$ direction in FI-MTJ and through the divergence of the spin current. The characteristic lengths of the torque oscillations are shown to be strongly dependent on the electronic and magnetic features of the FI, namely, the spin splittings of the two FI sublattices and the bandwidth parametrized here by the hopping matrix element of the TB Hamiltonian. Furthermore, we identify the fundamental parameter that governs the torque waves period in 1D FI-MTJ and the spatial extension of the damped torque oscillations in 3D FI-MTJ. These theoretical results may be of importance to choose the best-suited FI material for STT-based spintronic devices and will serve as a guideline for experiments on spin penetration length in these materials using, for example, ferromagnetic resonance and spin pumping effect $[20,21]$. In addition, experiments, such as spin-transfer-driven ferromagnetic resonance and spin-torque diode effect [22-25] where the magnitude and direction of the STT are extracted in magnetic tunnel junctions could also demonstrate the theoretical results predicted in this paper. 
[1] J. C. Slonczewski, J. Magn. Mater. 159, L1 (1996).

[2] L. Berger, Phys. Rev. B 54, 9353 (1996).

[3] S. A. Wolf, D. D. Awschalom, R. A. Buhrman, J. M. Daughton, S. von Molna, M. L. Roukes, A. Y. Chtchelkanova, and D. M. Treger, Science 294, 1488 (2001).

[4] A. H. MacDonald and M. Tsoi, Phil. Trans. R. Soc. A 369, 3098 (2011).

[5] A. Brataas, A. D. Kent, and H. Ohno, Nature Mater. 11, 372 (2012).

[6] M. Stamenova, S. Sanvito, and T. N. Todorov, Phys. Rev. B 72, 134407 (2005).

[7] S. Zhang and Z. Li, Phys. Rev. Lett. 93, 127204 (2004).

[8] A. Kalitsov, M. Chshiev, I. Theodonis, N. Kioussis, and W. H. Butler, Phys. Rev. B 79, 174416 (2009).

[9] E. M. Lifshitz, and L. P. Pitaievskii, Physical Kinetics, Course of Theoretical Physics (Pergamon, Oxford, 1981), Vol. 10.

[10] I. Theodonis, N. Kioussis, A. Kalitsov, M. Chshiev, and W. H. Butler, Phys. Rev. Lett. 97, 237205 (2006).

[11] Y.-H. Tang, N. Kioussis, A. Kalitsov, W. H. Butler, and R. Car, Phys. Rev. Lett. 103, 057206 (2009).

[12] M. D. Stiles and A. Zangwill, Phys. Rev. B 66, 014407 (2002).

[13] E. N. Economou, Green's Functions in Quantum Physics, Springer Series in Solid-State Sciences Vol. 7 (Springer, Berlin, Heidelberg, 2006).

[14] A. Kalitsov, I. Theodonis, N. Kioussis, M. Chshiev, W. H. Butler, and A. Vedyayev, J. Appl. Phys. 99, 08G501 (2006).
[15] D. M. Edwards, F. Federici, J. Mathon, and A. Umerski, Phys. Rev. B 71, 054407 (2005).

[16] M. A. Ruderman and C. Kittel, Phys. Rev. 96, 99 (1954); T. Kasuya, Prog. Theor. Phys. 16, 45 (1956); K. Yosida, Phys. Rev. 106, 893 (1957).

[17] A. S. Núñez, R. A. Duine, P. Haney, and A. H. MacDonald, Phys. Rev. B 73, 214426 (2006).

[18] A. Manchon, N. Ryzhanova, A. Vedyayev, M. Chschiev, and B. Dieny, J. Phys.: Condens. Matter 20, 145208 (2008).

[19] S. Wang, Y. Xu, and K. Xia, Phys. Rev. B 77, 184430 (2008).

[20] A. Ghosh, S. Auffret, U. Ebels, and W. E. Bailey, Phys. Rev. Lett. 109, 127202 (2012).

[21] P. Merodio, A. Ghosh, C. Lemonias, E. Gautier, U. Ebels, M. Chshiev, H. Béa, V. Baltz, and W. E. Bailey, Appl. Phys. Lett. 104, 032406 (2014).

[22] A. A. Tulapurkar, Y. Suzuki, A. Fukushima, H. Kubota, H. Maehara, K. Tsunekawa, D. D. Djayaprawira, N. Watanabe, and S. Yuasa, Nature (London) 438, 339 (2005).

[23] J. C. Sankey, P. M. Braganca, A. G. F. Garcia, I. N. Krivorotov, R. A. Buhrman, and D. C. Ralph, Phys. Rev. Lett. 96, 227601 (2006).

[24] J. C. Sankey Y.-T. Cui, J. Z. Sun, J. C. Slonczewski, R. A. Buhrman, and D. C. Ralph, Nat. Phys. 4, 67 (2008).

[25] H. Kubota, A. Fukushima, K. Yakushiji, T. Nagahama, S. Yuasa, K. Ando, H. Maehara, Y. Nagamine, K. Tsunekawa, D. D. Djayaprawira, N. Watanabe, and Y. Suzuki, Nat. Phys. 4, 37 (2008). 